\title{
Cross-Layer Combining of Adaptive Modulation and Truncated ARQ in Multichannel Beamforming MIMO Systems
}

\author{
Predrag IVANIS ${ }^{1}$, Dejan DRAJIC ${ }^{1,2}$, Srdan BRKIC ${ }^{1}$ \\ ${ }^{1}$ School of Electrical Engineering, University of Belgrade, Bulevar kralja Aleksandra, 73, 11000 Belgrade, Serbia \\ 2 IRITEL A.D., Batajnicki drum 23, Belgrade, Serbia \\ predrag.ivanis@etf.rs, ddrajic@etf.rs, srdjan.brkic@ic.etf.rs
}

\begin{abstract}
In this study the authors provide a cross-layer design of multiple-input-multiple-output (MIMO) systems, with the aim to maximize spectral efficiency. We consider MIMO systems based on a multichannel beamforming technique that combines an adaptive modulation and truncated automatic repeat request procedures, for the case of Rayleigh fading propagation and imperfect channel state information. Closed-form expressions for the average spectral efficiency and the packet loss rate are derived for arbitrary eigenchannel of multichannel beamforming systems, with any number of receiving and transmitting antennas. An analytical expression for the average time during which a particular constellation is used continuously, is also derived. We propose the method based on the optimization of the target packet error rate and the maximum number of retransmissions that outperforms the existing cross-layer combining procedures. Furthermore, we develop the numerical algorithm for optimization of the eigenchannel power allocation. The proposed cross-layer design results in higher average spectral efficiency, reduced maximum delay and increased energy efficiency. The analytical results are validated by Monte Carlo simulation.
\end{abstract}

\section{Keywords}

Adaptive modulation, automatic repeat request, crosslayer design, MIMO systems, Rayleigh channels.

\section{Introduction}

It is well known that a multiple-input-multiple-output (MIMO) channel can be transformed into mutually separated eigenchannels, when channel state information (CSI) is known at the transmitter and the receiver $[1,2]$. Singular value decomposition (SVD) combined with the adaptive modulation and appropriate power allocation enable significant increase of the average spectral efficiency (ASE), in comparison to single-input-single-output (SISO) systems [35]. However, when all eigenchannels are used the overall error performance of the corresponding system is dominated by the weakest eigenchannel (with the power gain determined with the minimum eigenvalue of the corresponding Wishart matrix) [5].

The multilevel modulation combined with the increased diversity order results in the increase of the throughput available to the user, while the challenging task remains to chose the diversity technique that provides reasonable complexity [6]. The complexity of MIMO systems can be reduced, at the cost of the decreased ASE, if only the strongest eigenchannel is used for data transfer [7]. This solution, known as beamforming, is efficient even when CSI is not perfectly known at the transmitter and the receiver [8,9]. The increase of the throughput is possible if only a subset of available eigenchannels is used for the transmission. Performance analysis of a such system, referred to as MIMO multichannel beamforming (MIMO-MB), is possible if statistical properties of ordered eigenvalues of the corresponding Wishart matrix are available [10, 11].

On the other hand, using the joint optimization of the adaptive modulation at the physical layer and the automatic repeat request $(\mathrm{ARQ})$ at the data link layer, it is possible to maximize the ASE, under predefined delay and packet error performance constraints. Cross-layer combining of adaptive modulation (AM) with truncated selective repeating (SR) ARQ in SISO systems is proposed in [12], for the case when quality of service (QoS) is determined by the probability of packet loss. Using the same approach, the ASE of spacetime coded MIMO systems with perfect CSI is determined [13]. An iterative algorithm for finding the optimal switching thresholds for attaining maximum spectral efficiency in MIMO systems that combines AM and ARQ is proposed in [14]. Performance of cross-layer design in multiuser MIMO Systems, in the presence of outdated estimates, is presented in [15]. The overview of various cross-layer mechanisms and its applications in wireless networks is given in [16].

The cross-layer approaches for the transmission through the eigenchannels are analyzed in [17] for the Rayleigh fading environment, but the approximate expressions for the eigenvalue distributions are used to estimate the error rates and throughputs. Exact expressions for the bit error rate (BER) and the ASE for arbitrary constellation sizes are derived in 
[18]. However, in that paper only the case of two eigenchannels is investigated, but adaptation of the constellation size was not considered.

In this paper we propose link adaptation in MIMO-MB systems for the predefined probability of packet loss. For the proposed method that combines the adaptive modulation, the truncated SR-ARQ and the eigenchannel power allocation, the relevant performance measures are derived. The main contributions of the paper are listed below.

- We extend the analysis given in [12] to the eigenchannels of MIMO-MB systems. Using the exact Probability Density Function (PDF) expressions for the instantaneous signal-to-noise ratio (SNR) in an eigenchannel, we derive the expressions for the average packet error rate (PER) and the ASE for this more general case. For any eigenchannel we derive the exact expressions for the probability of the transmission mode choice and the average time of its continuous usage.

- The cross-layer design method proposed in [12] is extended to every particular eigenchannel of MIMO-MB systems with arbitrary system dimensions. It will be shown that target the PER at the physical layer that provide maximum tolerable packet loss rate does not guarantee the maximal spectral efficiency. Also, it will be shown that additional retransmissions do not necessary increase the ASE when the target PER is chosen to preserve maximum tolerable packet loss rate at the fixed level, as it was incorrectly assumed in [12]. Therefore, the method for joint optimization of the target PER and the number of retransmissions in the SR-ARQ procedure is proposed, under the constraints that the packet loss rate and tolerated delay are limited.

- Finally, we perform the joint optimization of the target PER, the number of retransmissions and the eigenchannel power allocation coefficients in order to maximize ASE of MIMO-MB systems with the imperfect CSI knowledge, under the prescribed packet loss rate level.

\section{Channel and System Model}

We consider a single-user MIMO system employing $n_{T}$ antennas for the transmission and $n_{R}$ antennas for the reception. A received vector, denoted by $\mathbf{y}$, is given by [1]

$$
\mathbf{y}=\mathbf{H x}+\mathbf{n},
$$

where $\mathbf{H}$ is a $\left(n_{R} \times n_{T}\right)$ channel matrix, $\mathbf{x}$ represents a $\left(n_{T} \times 1\right)$ vector of the symbols sent from the transmitting antennas, and a $\left(n_{R} \times 1\right)$ additive noise vector is denoted by $\mathbf{n}$.

We assume frequency flat fading and that the entries of the channel matrix are mutually independent complex zero-mean unit-variance Gaussian random variables (GRVs). Every entry of the noise vector is zero-mean, normally distributed with variance $\sigma_{n}^{2}$, and the average SNR is defined as the ratio of the total transmit power (denoted by $P$ ) and the noise power per receiving antenna, i.e. $\bar{\gamma}=P / \sigma_{n}^{2}$.

The CSI model is adopted from [3, 4], where the identical CSI is known to the transmitter and the receiver, and imperfect CSI is represented by a channel matrix $\hat{\mathbf{H}}$, which is related to the true channel matrix $\mathbf{H}$ as

$$
\mathbf{H}=\hat{\mathbf{H}}+\Xi,
$$

where $\Xi$ denotes an estimation error matrix. The entries and $\hat{\mathbf{H}}$ are independent complex zero-mean GRVs, with variances $\sigma_{e}^{2}$, and $1-\sigma_{e}{ }^{2}$, respectively [3, 8]. Using the SVD theorem, the estimated channel matrix $\hat{\mathbf{H}}$ can be written in the form $\hat{\mathbf{H}}=\hat{\mathbf{U}} \hat{\mathbf{D}} \hat{\mathbf{V}}^{H}$, where $\hat{\mathbf{U}}$ and $\hat{\mathbf{V}}$ are unitary matrices and superscript $H$ denotes the Hermitian transpose operator. Entries of diagonal matrix $\hat{\mathbf{D}}$ are the square roots of the matrix $\hat{\mathbf{W}}=\hat{\mathbf{H}} \hat{\mathbf{H}}^{H}$ eigenvalues, denoted by $\hat{\lambda}_{n}, 1 \leq n \leq M$, where $M$ denotes minimum of $n_{R}$ and $n_{T}$.

According to [1], the optimal transmit and receive weight vectors for the $n$th eigenchannel are determined with the eigenvectors (columns of $\hat{\mathbf{V}}$ and $\hat{\mathbf{U}}^{H}$ ) that correspond to the $n$th eigenvalue of the matrix $\hat{\mathbf{W}}$, as it is shown in Fig. 1 . The power gain of the $n$th eigenchannel can be represented as $\hat{\lambda}_{n}=\lambda_{n} /\left(1-\sigma_{e}^{2}\right)$, where $\lambda_{n}$ denotes the $n$th eigenvalue of the Wishart matrix $\mathbf{W}=\mathbf{H H}^{H}$ [8, Eq. 41]. If we combine it with [4, Eq. 56], the expression for the SNR at the $n$th eigenchannel output is obtained as

$$
\gamma_{n}=\lambda_{n} \times \frac{\alpha_{n} \bar{\gamma}}{\left(1-\sigma_{e}^{2}\right)\left(1+\alpha_{n} \bar{\gamma} \sigma_{e}^{2}\right)}=\lambda_{n} \times \bar{\gamma}_{n}^{e q},
$$

where $\bar{\gamma}_{n}^{e q}$ denotes the equivalent average SNR in the $n$th eigenchannel, that includes the effect of the crosseigenchannel interference. In the above equation, $\alpha_{n}$ denotes a normalized power allocation coefficient that determines the portion of the power transmitted through the $n$th eigenchannel and satisfies $\sum_{k=1}^{M} \alpha_{n}=1$.

The modulator for the $n$th eigenchannel is located prior to the transmit weight vector, as it is shown in Fig. 1. We adopted the $L$ quadrature amplitude modulation (QAM) constellations with size $2^{m}, m=1,2, \ldots L$.

Depending on the instantaneous eigenchannel gain and the average SNR in the channel, the transmitter chooses the constellation with the maximal size that satisfies a predefined error constraint. As it was usually considered [12, 13], when constellation with $2^{m}$ points is chosen, the train of $R_{m}=m$ packets is modulated into the frame with $N_{F}$ symbols. Each packet contains overhead, payload, and cyclic redundancy check (CRC) bits to facilitate error detection. At the beginning of every frame, we also add pilot symbols for estimation and $N_{C}$ symbols that carry out information about the chosen modulation format.

Although the instantaneous packet error rate can be calculated from the above equation, as it was shown in [12], for the typical values of PER it can be accurately represented as [12, Eq. 5] 


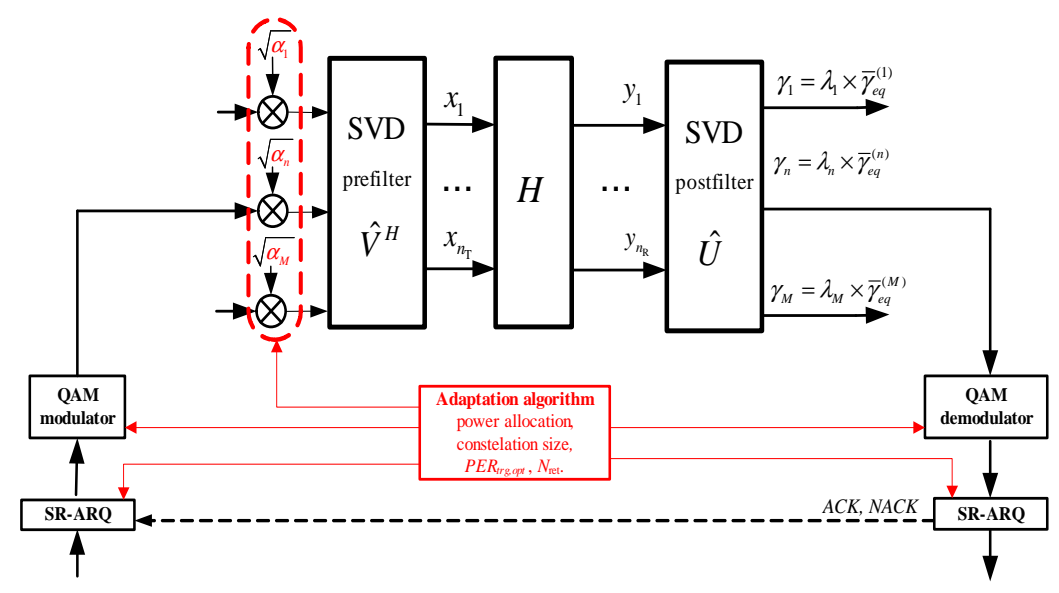

Fig. 1. SVD-based transmission with QAM and SR-ARQ.

\begin{tabular}{|c|c|c|c|c|c|c|c|}
\hline Modulation & BPSK & QPSK & 8-QAM & 16-QAM & 32-QAM & 64-QAM & 128-QAM \\
\hline$R_{m}[\mathrm{bit} / \mathrm{s}]$ & 1 & 2 & 3 & 4 & 5 & 6 & 7 \\
\hline$a_{m}$ & 67.733 & 73.828 & 58.733 & 55.914 & 50.055 & 42.559 & 40.256 \\
\hline$g_{m}$ & 0.982 & 0.459 & 0.164 & 0.099 & 0.038 & 0.024 & 0.009 \\
\hline$\gamma_{p m}[\mathrm{~dB}]$ & 6.328 & 9.395 & 13.947 & 16.094 & 20.110 & 22.034 & 25.968 \\
\hline
\end{tabular}

Tab. 1. Transmission modes and parameters of exponential approximation.

$$
P E R_{m}^{(n)}\left(\gamma_{n}\right)= \begin{cases}1, & 0 \leq \gamma_{n} \leq \gamma_{p m} \\ a_{m} \exp \left(-g_{m} \gamma_{n}\right), & \gamma_{n}>\gamma_{p m}\end{cases}
$$

where $\gamma_{n}$ is a instantaneous SNR at the $n$th eigenchannel output. Parameters $a_{m}, b_{m}$ and $\gamma_{p m}$ are mode-dependent, and are obtained by fitting the above expression to the exact PER. The values presented in Tab. 1 are obtained for $N_{F}=1080$ and they are taken from [12]. When we use the adaptive modulation, at the beginning of every transmission frame we choose a modulation format that enables the highest spectral efficiency and also satisfies the target packet error rate (at the physical layer) that corresponds to the $n$th eigenchannel, denoted by $\operatorname{PER}_{\text {trg }}^{(n)}$. In particular, we choose the constellation that results in the instantaneous SNR above the corresponding threshold defined as

$$
\Gamma_{m}^{(n)}= \begin{cases}-\ln \left(\mathrm{PER}_{\mathrm{trg}}^{(n)} / a_{m}\right) / g_{m}, & m=1,2, \ldots, N_{m}, \\ 0, & m=0,\end{cases}
$$

and if $\Gamma_{0}^{(n)}<\gamma_{n}<\Gamma_{1}^{(n)}$, the payload transmission is blocked, but the overhead symbols are transmitted.

At the data link layer, the receiver performs CRC check and sends positive or negative acknowledgment (ACK/NACK) for every packet through the feedback channel to the transmitter (that performs retransmission if it is necessary). As in [12], we also considered the truncated selective repeating ARQ procedures with the maximal number of retransmissions $N_{\text {ret }}$. However, we did not apply standard method for obtaining the target PER from the predefined probability of packet loss rate, proposed in [12]. As we will show in the next section, our optimization method results in higher ASE and the reduced maximum delay, especially for higher values of the average SNR.

We list all the assumptions we have used:
AS1) The fading is frequency flat and slow, remains nearly constant during one frame, but varies from a frame to a frame. The fading channel coefficients that correspond to the retransmitted copies of a packet are described using independent random variables. These conditions are satisfied when a frame duration is smaller than the channel coherence time and the round trip delay (RTD) is much larger than the channel coherence time.

AS2) Error detection based on the CRC is perfect and overhead bits in each packet are not included in the throughput calculation, because they introduce negligible redundancy relative to the payload [12-14].

Furthermore, we assume that the power allocation coefficients do not vary in time, but their values depend on the average SNR. It can be noticed that this approach is not optimal since the optimal power allocation is proposed in [4] and represents the extension of temporal water-filling method to the adaptive modulation systems. In the variablerate variable-power approach it is assumed that power allocation is adapted to the current eigenvalues realization, with the constraint that the average transmitted power is constant. This method requires the fast power adaptation and, therefore, has large implementation complexity. Furthermore, during the deep fades the required peak power values (obtained as a result of the adaptation process) can be very high. In contrary, we want to determine the optimal power allocation coefficients for a given average SNR with the constant instantaneous power transmitted from antennas. This approach includes the important special cases - when $\alpha_{i}=1$ and $\alpha_{n}=0$ for $n>1$, we have beamforming system [7-9], and when $\alpha_{n}=\alpha_{2} \ldots=\alpha_{M}=1 / M$ the power allocation is uniform, i.e., it is the variable rate mode analyzed in $[3,4]$. 


\section{Performance Analysis}

The exact PDF expression for the $n$th largest eigenvalue of the $M \times M$ Wishart matrix is given in [19], for the case of Rayleigh propagation environment

$$
f_{\lambda_{n}}\left(\lambda_{n}\right)=\sum_{z=1}^{Z_{\max }} A_{z}^{(n)} \lambda_{n}^{B_{z}^{(n)}} \exp \left(-C_{z}^{(n)} \lambda_{n}\right)
$$

where the summation in above expression is defined as

$$
\sum_{z=1}^{Z_{\max }}=\sum_{s=n}^{M} \sum_{\alpha} \sum_{\mu} \sum_{t_{1}=0}^{N-M+\mu_{1}-1} \sum_{t_{2}=0}^{N-M+\mu_{2}-1} \cdots \sum_{t_{s-1}=0}^{N-M+\mu_{s-1}-1},
$$

and $\alpha$ and $\mu$ denote complete sets of permutations of vector $(1,2, \ldots, M)$, while $Z_{\text {max }}$ denotes the total number of terms in the multiple sum on the right hand side of the above identity. The corresponding coefficients are defined as

$$
\begin{aligned}
& A_{z}^{(n)}=\frac{s(-1)^{s-n} \operatorname{sgn}(\alpha) \operatorname{sgn}(\mu)}{M ! \prod_{i=1}^{M}(N-i) ! \prod_{j=1}^{M}(M-j) !}\left(\begin{array}{c}
s-1 \\
n-1
\end{array}\right)\left(\begin{array}{c}
M \\
s
\end{array}\right) \\
& \times \prod_{l=s+1}^{M}\left(N-M+\alpha_{l}+\mu_{l}-2\right) ! \prod_{l=1}^{s-1} \frac{\left(N-M+\alpha_{l}+\mu_{l}-2\right) !}{t_{l} !},
\end{aligned}
$$

$B_{z}^{(n)}=N-M+\alpha_{s}+\mu_{s}-2+\sum_{l=1}^{s-1} t_{l}, C_{z}^{(n)}=s$, and $\alpha_{s}$, $\mu_{s}$ denotes the $s$ th symbol in the observed permutation.

The PDF of the instantaneous SNR at the output of the $n$th eigenchannel are then obtained by using expression (3) and relations [20, Eqs. (6.5.2), (6.5.13)]

$$
f_{\gamma_{n}}\left(\gamma_{n}\right)=\frac{1}{\bar{\gamma}_{n}^{e q}} \sum_{z=1}^{Z_{\max }} A_{z}^{(n)}\left(\frac{\gamma_{n}}{\bar{\gamma}_{n}^{e q}}\right)^{B_{z}^{(n)}} \exp \left(-\frac{C_{z}^{(n)} \gamma_{n}}{\bar{\gamma}_{n}^{e q}}\right)
$$

and the probability of choosing the $m$ th transmission mode is derived as

$$
\begin{aligned}
\pi_{m}^{(n)} & =\int_{\Gamma_{m}^{(n)}}^{\Gamma_{m+1}^{(n)}} f_{\gamma_{n}}\left(\gamma_{n}\right) d \gamma_{n}=\sum_{z=1}^{Z_{\max }} \frac{A_{z}^{(n)}\left(B_{z}^{(n)} !\right)}{\left(C_{z}^{(n)}\right)^{B_{z}^{(n)}+1}} \sum_{l=0}^{B_{z}^{(n)}} \frac{1}{l !} \\
& \times\left[\left(C_{z}^{(n)} \frac{\Gamma_{m}^{(n)}}{\bar{\gamma}_{n}^{e q}}\right)^{l} \exp \left(-C_{z}^{(n)} \frac{\Gamma_{m}^{(n)}}{\bar{\gamma}_{n}^{e q}}\right)\right. \\
& \left.-\left(C_{z}^{(n)} \frac{\Gamma_{m+1}^{(n)}}{\bar{\gamma}_{n}^{e q}}\right)^{l} \exp \left(-C_{z}^{(n)} \frac{\Gamma_{m+1}^{(n)}}{\bar{\gamma}_{n}^{e q}}\right)\right] .
\end{aligned}
$$

Furthermore, we derive the average PER for the $m$ th mode and the $n$th eigenchannel by using the approach from [12], substitution $b_{m}=C_{z}^{(n)} / \bar{\gamma}_{n}^{e q}+g_{m}$ and by applying relations [20, Eqs. (6.5.2), (6.5.13)] to obtain

$$
\begin{aligned}
& \overline{\operatorname{PER}}_{m}^{(n)}=\frac{1}{\pi_{m}^{(n)}} \sum_{z=1}^{Z_{\max }} \frac{a_{m} A_{z}^{(n)}\left(B_{z}^{(n)} !\right)}{\left(b_{m} \bar{\gamma}_{n}^{e q}\right)^{k_{2, z}^{(n)}+1}} \sum_{l=0}^{B_{z}^{(n)}} \frac{1}{l !} \\
& \times\left[\left(b_{m} \Gamma_{m}^{(n)}\right)^{l} \exp \left(-b_{m} \Gamma_{m}^{(n)}\right)-\left(b_{m} \Gamma_{m+1}^{(n)}\right)^{l} \exp \left(-b_{m} \Gamma_{m+1}^{(n)}\right)\right] .
\end{aligned}
$$

For the special case of $M=N=1$ and the perfect CSI we obtain $Z_{\text {max }}=1$ with $\bar{\gamma}_{n}^{e q}=\bar{\gamma}, A_{1}^{(1)}=1, B_{1}^{(1)}=0, C_{1}^{(1)}=1$, $b_{m}=1 / \bar{\gamma}+g_{m}$ and Eqs. (10) and (11) respectively reduce to [12, Eqs. (7)] and [12, Eqs. (8)].

Following the procedure from [12], we derive the expressions for the actually obtained average PER in the $n$th eigenchannel (that is always less than the target value $\mathrm{PER}_{\mathrm{trg}}^{(n)}$ and the packet loss rate after $N_{\text {ret }}$ retransmissions

$$
\begin{gathered}
\overline{\mathrm{PER}}^{(n)}=\sum_{m=1}^{N_{m}} R_{m} \pi_{m}^{(n)} \overline{\mathrm{PER}}_{m}^{(n)} / \sum_{m=1}^{N_{m}} R_{m} \pi_{m}^{(n)}, \\
p_{\text {loss }}^{(n)}=\left(\overline{\mathrm{PER}}^{(n)}\right)^{N_{\mathrm{ret}}+1},
\end{gathered}
$$

and the overall ASE after $N_{\text {ret }}$ retransmissions is obtained as a summation of ASEs in the separate eigenchannels

$$
\eta_{N_{\mathrm{ret}}}^{T \text { OT }}=\sum_{n=1}^{M} \eta_{N_{\mathrm{ret}}}^{(n)}=\sum_{n=1}^{M} \frac{1-\overline{\mathrm{PER}}^{(n)}}{1-\left(\overline{\mathrm{PER}}^{(n)}\right)^{N_{\text {ret }}+1}} \sum_{m=1}^{N_{m}} R_{m} \pi_{m}^{(n)} .
$$

The average time during which a particular constellation is continuously used is given by [19, Eq. (34)]

$$
T_{m}=\pi_{m}^{(n)} /\left(N_{\Gamma_{m+1}^{(n)}}-N_{\Gamma_{m}^{(n)}}\right)
$$

where $N_{\Gamma_{m}^{(n)}}$ denotes the level crossing rate (LCR) for the instantaneous SNR in the $n$th eigenchannel and level $\Gamma_{m}^{(n)}$.

Combining the above identity with the LCR expression previously obtained in [11, Eq. 12], we derive the average time during which a particular constellation is continuously used in the $n$th eigenchannel of the MIMO-MB system as

$$
\begin{aligned}
& T_{m}=\frac{1}{f_{\mathrm{m}}} \sqrt{\frac{\left(1-\sigma_{e}^{2}\right)\left(1+\alpha_{n} \bar{\gamma} \sigma_{e}^{2}\right)}{2 \pi \alpha_{n} \bar{\gamma}}} \\
& \times \frac{\pi_{m}^{(n)}}{\left(\sqrt{\Gamma_{m+1}^{(n)}} f_{\gamma_{n}}\left(\Gamma_{m+1}^{(n)}\right)+\sqrt{\Gamma_{m}^{(n)}} f_{\gamma_{n}}\left(\Gamma_{m}^{(n)}\right)\right)}, m=1,2, \ldots, N_{m}
\end{aligned}
$$

that can be easily expressed in a closed-form expression by using (9) and (10). 


\section{The Optimization Procedure}

\subsection{Optimization of the PER}

In the previous section we have derived the expressions for the ASE, the packet loss rate and the average time during which a particular constellation is continuously used. It can be noticed that the corresponding numerical results for every particular eigenchannel can be obtained only if we know the average SNR, variance of the CSI imperfection, the maximal number of retransmissions, the portion of the power allocated to the eigenchannel and SNR thresholds that correspond to the particular constellations, respectively denoted by $\bar{\gamma}, \sigma_{e}^{2}$, $N_{\text {ret }}, \alpha_{n}$ and $\Gamma_{0}^{(n)}, \Gamma_{1}^{(n)}, \ldots, \Gamma_{N_{m}}^{(n)}$. As it has been shown in (5), the SNR thresholds depend on the value of $\mathrm{PER}_{\operatorname{trg}}^{(n)}$, that has to be chosen at the physical layer to ensure a QoS constraint. On the other hand, the parameter $N_{\text {ret }}$ needs to be bound in the data link layer to ensure a delay constraint. In this section, we consider the case when only the $n$th eigenchannel is active $\left(\alpha_{n}=1\right)$ and we show that it is possible to determine the optimal combination of $\mathrm{PER}_{\text {trg, opt }}^{(n)}$ and $N_{\text {ret }}$ that results in the highest attainable spectral efficiency and reduced delay when compared to the existing methods, for the fixed value of the target packet loss rate.

In standard method, that was previously used in [12-15], the target PER was calculated as

$$
\operatorname{PER}_{\text {trg, std }}^{(n)}=\left(p_{\text {loss, trg }}\right)^{\frac{1}{N_{\text {ret }}+1}},
$$

where $p_{\text {loss,trg }}$ denotes the predetermined target packet loss rate.

As it was already noticed in [12], this criterion have important drawback. Due to the discrete size of constellations, relation $p^{(n)}<\operatorname{PER}_{\text {trg, std }}^{(n)}$ always holds, and the actual values for packet loss rate (calculated by expression (13)) are

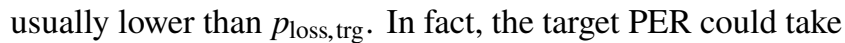
greater values then it is determined by expression (17) and the actual packet loss rate still remains below the target value for the packet loss rate.

Furthermore, it does not mean that the target packet error rate that ensures packet error loss rate to be equal to $p_{\text {loss, trg }}$ produces the maximal spectral efficiency. We show that, if the maximum of a function $\eta_{N_{\text {ret }}}^{(n)}\left(\mathrm{PER}_{\mathrm{trg}}^{(n)}\right)$ is located in a region where $p_{\text {loss }}<p_{\text {loss, trg }}$, the spectral efficiency can be increased additionally using the joint optimization of the target packet error rate and $N_{\text {ret }}$, for the fixed average SNR. If we assume that $\mathrm{PER}_{\text {trg }}^{(n)}$ is fixed, the actual packet error rate at the physical layer is determined by (12). In such a case, it is obvious from expression (14) that the ASE actually can not be further enlarged by increasing the maximum number of retransmissions, i.e. for any fixed value of $\mathrm{PER}_{\mathrm{trg}}$, the average spectral efficiency decreases when $N_{\text {ret }}$ increases.

If we determine $\operatorname{PER}_{\text {trg, opt }}^{(n), N_{\text {ret }}}$ that maximizes spectral efficiency for fixed $N_{\text {ret_1 }}$, even a higher value for ASE can be achieved for $N_{\text {ret_2 }}<N_{\text {ret_1 }}$ for the unchanged target packet error rate. We can also notice that the optimal value of target PER that maximizes the average spectral efficiency for $N_{\text {ret_2 }}$ retransmissions has different value, denoted as $\mathrm{PER}_{\mathrm{trg}, \mathrm{opt}}^{(n), 2}$, and therefore

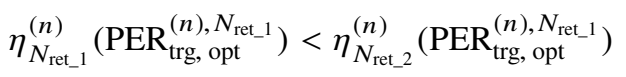

$$
\begin{aligned}
& <\eta_{N_{\text {ret_2 }}}^{(n)}\left(\mathrm{PER}_{\mathrm{trg}, \mathrm{opt}}^{\left.(n), N_{\text {ret } 2}\right) .}\right.
\end{aligned}
$$

In addition, the QoS constraint remains satisfied only if the packet loss rate that corresponds to $\operatorname{PER}_{\mathrm{trg}, \text { opt }}^{(n), N_{\mathrm{ret}, 2}}$ is below the predefined target value. If this condition is satisfied for reduced number of transmissions, the spectral efficiency can be further increased if the appropriate target packet error rate is chosen for this case. This effect is somewhat surprising and it is in contrast with the conclusions of the paper [12], that the spectral efficiency is always increased for the higher number of retransmissions. Ostensible increase, described in [12], is the result of non optimal selection of the target PER. To the best of the authors' knowledge, this effect has not been noticed before. Therefore, we anticipate that the best results will be obtained if we choose the minimum value for $N_{\text {ret }}$ and the corresponding optimal value $\mathrm{PER}_{\mathrm{trg}}^{(\mathrm{n}), \mathrm{N}_{\mathrm{ret}}}$ that maximizes the ASE in the $n$th eigenchannel under a constraint $p_{\text {loss }} \leq p_{\text {loss, }}$ trg.

\subsection{Joint Optimization Procedure}

In the previous section we have provided the basic elements for an evaluation procedure of the target PER that maximizes the average spectral efficiency in the $n$th eigenchannel of MIMO-MB system. For the fixed number of retransmissions $N_{\text {ret }}$, if the corresponding ASE curve has a maximum in region where $p_{\text {loss }}^{(n)} \leq p_{\text {loss, trg }}$, we can use the following criterion

$$
\partial \eta_{N_{\text {ret }}}^{(n)} / \partial \operatorname{PER}_{\mathrm{trg}, \text { opt }}^{(n), N_{\text {ret }}}=0
$$

and otherwise the optimal PER is the one that satisfies $p_{\text {loss }}^{(n)}=p_{\text {loss, trg }}$. The derivation of the spectral efficiency function can be done analytically, using Leibniz's rule and the $\mathrm{PER}_{\mathrm{trg}}^{(\mathrm{n}), \mathrm{N}_{\mathrm{ret}}}$ can be found accurately applying the augmented Lagrange multiplier method [22].

However, for the case when more than one eigenchannel is active we can find the optimal $\operatorname{PER}_{\text {trg, opt }}^{(n), N_{\text {ret }}}$ and the power allocation coefficient for every particular eigenchannel. In such a case, determining the exact values of optimal parameters is not a simple task and more reasonable solution is to use a numerical constraint optimization algorithm. Thus, if $M$ eigenchannels are used for the transmission it is necessary to solve $2 M$-dimensional optimization problem: $M$ optimal values of eigenchannel power allocation coefficients and $M$ optimal values for the target PERs in the eigenchannels have to be obtained.

If we define a constraint optimization problem as follows 


$$
\max : \eta_{N_{\text {ret }}}^{\mathrm{TOT}}\left(\mathrm{PER}_{\mathrm{trg}}^{(1)}, \ldots, \mathrm{PER}_{\mathrm{trg}}^{(M)}, \alpha_{1}, \ldots, \alpha_{M}\right)
$$

subject to:

$$
\begin{aligned}
& p_{\text {loss }}^{(n)} \leq p_{\text {loss, trg }}^{(n)}, n=1,2, \ldots, M, \\
& 0 \leq \mathrm{PER}_{\operatorname{trg}}^{(n)} \leq 1, n=1,2, \ldots, M, \\
& \sum_{n=1}^{M} \alpha_{n}=1,
\end{aligned}
$$

we can use the Sequential Quadratic Programming (SQP) optimization algorithm to find a local maximum of the function $\eta\left(\mathrm{PER}_{\mathrm{trg}}^{(1)}, \ldots, \mathrm{PER}_{\mathrm{trg}}^{(n)}, \alpha_{1}, \ldots, \alpha_{n}\right)$. The procedure is repeated for integer values of parameter $N_{\text {ret }}<N_{\text {ret, max }}$ and a set of optimization parameters that produces the maximal ASE is chosen. A brief description of the SQP method that we used to obtain the numerical results is presented in Appendix.

\section{Numerical Results}

In this section, we present numerical results for the average spectral efficiency when the proposed optimization procedure is applied and it is compared with the results obtained by using the standard cross-layer method. We consider the case of $N_{F}=1080$ information symbols and the frame duration is typically $T_{F}=2 \mathrm{~ms}$ [12]. We assume typical carrier frequency $f_{0}=2.4 \mathrm{GHz}$, pedestrian speed, and the coherence time $T_{c o h}=38 \mathrm{~ms}$. As round trip delay is typically greater than $\tau=100 \mathrm{~ms}$, it is obvious that the assumption AS1 (defined in Sec. 2) is reasonable. As the tolerated delay is typically $300-400 \mathrm{~ms}$ [12], we assumed the maximal number of retransmissions $N_{\text {ret }} \leq N_{\text {ret, } \max }=3$.

The numerical results obtained by the analytical approach are validated by using Monte Carlo simulations. Sequences of $L=10^{7}$ fading samples are generated for every channel coefficient using the modified Jakes simulator [23]. We estimated $L$ successive realizations of the matrix $\hat{\mathbf{H}}$ and applied the SVD algorithm to obtain the sequence of the ordered eigenvalues. As we assumed that the sampling interval is equal to the frame duration, we obtained the instantaneous SNRs for $L$ successive frames in every particular eigenchannel by using (3). For every transmitted frame, the optimum constellation size is determined, the noise is added and the demodulation is performed [24]. The erroneous packets are retransmitted with the round trip delay equal to the duration of $\tau / T_{F}=50$ frames and the ASE is estimated by dividing the total number of transmitted information bits with the total number of signalization intervals.

As an example for the optimization of the target PER and $N_{\text {ret }}$, we consider the beamforming system $\left(n=1, \alpha_{1}=1\right)$ with $N=M=2$ and $\bar{\gamma}=15 \mathrm{~dB}$. For the target packet error rate in the range $[0.01,1]$, the ASE and the $p_{\text {loss }}$ values obtained from (9)-(14) are presented in Figs. 2 and 3. We can observe from Fig. 2 that the value of ASE for the case with $N_{\text {ret }}=1$ is higher than in the case $N_{\text {ret }}=3$ for

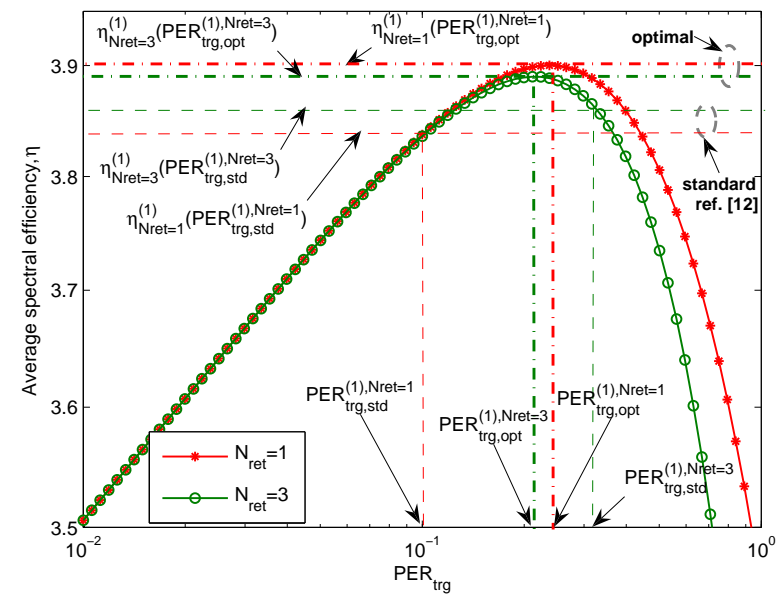

Fig. 2. Average spectral efficiency for various target PERs and number of retransmissions, $p_{\text {loss, }}$ trg $=0.01$.

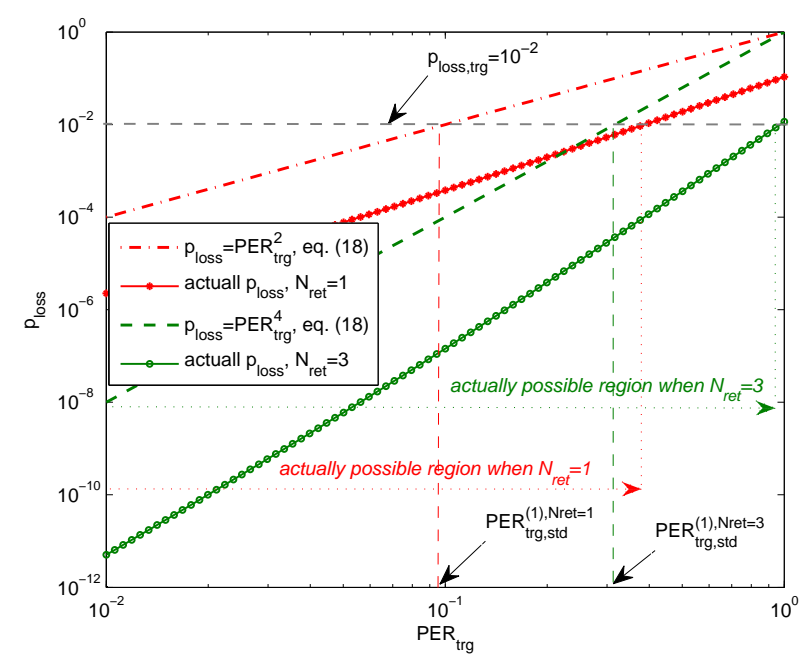

Fig. 3. Assumed and actual packet loss rates for various target PERs and number of retransmissions, $p_{\text {loss, } \operatorname{trg}}=0.01$.

any $\operatorname{PER}_{\text {trg }}^{(1)}$, as explained in Sec. 4. The target packet error rate obtained from (17) results in the packet loss rate lower than the desired value ( $p_{\text {loss, } \operatorname{trg}}=0.01$ ), but it is not optimal. To reach the maximal ASE for the case of $N_{\text {ret }}=3$, $\operatorname{PER}_{\text {trg, opt }}^{(1), N_{\text {ret }}=3}<\operatorname{PER}_{\text {trg, std }}^{(1), N_{\text {ret }}=3}$ has to be chosen. The spectral efficiency can be further increased if the maximal number of retransmissions is reduced to $N_{\text {ret }}=1$, and $\operatorname{PER}_{\text {tro, opt }}^{(1), N_{\text {ret }}=1}$ has to be recalculated for this case. As the actual packet loss rate is lower than the target value $p_{\text {loss, trg }}$ even when the number of retransmissions is reduced, solution with $N_{\text {ret }}=1$ and optimized value for PER $\mathrm{trg}_{\mathrm{tr}}^{(1)}$ that satisfies QoS conditions, results in the higher ASE and therefore outperforms the standard solution with $N_{\text {ret }}=3$ retransmissions.

Optimized beamforming system is compared with the case where the target PER was chosen according to [12] and the corresponding spectral efficiencies are presented in Fig. 4. The improvements achieved by the proposed optimization method are noticeable in the whole SNR range for 


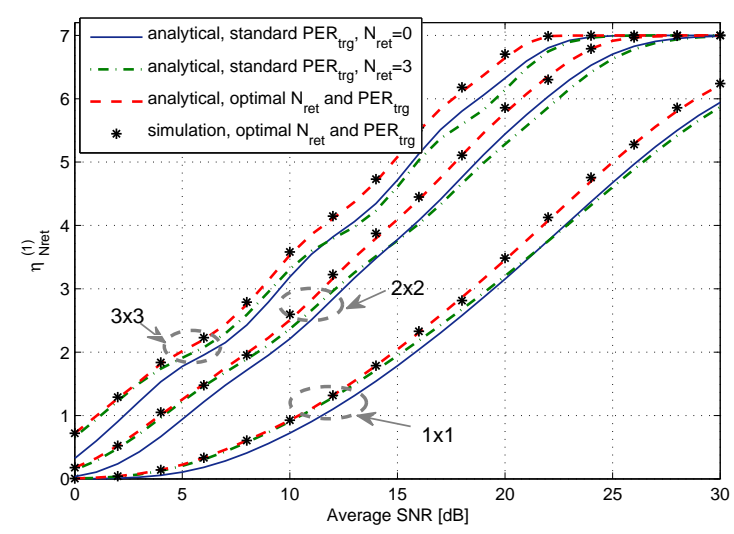

Fig. 4. Average spectral efficiency for the beamforming system, various system dimensions, $p_{\text {loss, trg }}=0.05$, optimal power allocation and optimal chose of PER trg $_{\text {and }} N_{\text {ret }}$.

all analyzed system dimensions. The figure also illustrates that, in this particular case, the spectral efficiency for the higher SNR is larger for the case without retransmissions $\left(N_{\text {ret }}=0\right)$ than for the case with $N_{\text {ret }}=3$, even when it is calculated using the standard approach proposed in [12]. Therefore, as we jointly optimize the target PER and $N_{\text {ret }}$, we anticipate that the optimal values for $N_{\text {ret }}$ have to be smaller for the high SNR region. The optimal $N_{\text {ret }}$ for different SNR ranges and two values of the desired packet loss rate are determined and given in Tab. 2. For a case of the SISO system and lower values of $p_{\text {loss, }}$ trg, the large maximum number of retransmissions is optimal for the wide SNR range that verifies the practical conclusions of [12] and also corresponds to the results presented in [12, Fig. 8], where it is concluded that the larger $N_{\text {ret }}$ is more appropriate when target $p_{\text {loss, trg }}$ has lower value. However, even for rigorous QoS requirements, we noticed that the ASE can be further maximized in the high average $\mathrm{SNR}$ region if we optimize $P E R_{\mathrm{trg}}$ and reduce $N_{\text {ret }}$. When diversity order $(M \times N)$ increases, the smaller number of retransmissions results in the greater ASE even for moderate values of the average SNR. Maximal number of retransmissions can be reduced without negative impacts to QoS requirements and therefore the proposed optimization procedure results in a reduced maximal delay in the packet delivery comparing to the standard cross-layer procedure [12].

The average spectral efficiency for the case of MIMOMB with three antennas at the transmitter and the receiver and optimal power allocation is presented in Fig. 5. The results are compared to the system with only one active eigenchannel $\left(\alpha_{1}=1\right)$ and to the system with the uniform power allocation, both with optimized values of the target PERs and the number of retransmissions. For very small SNR values, it is optimal to transfer data only through the strongest eigenchannel. However, for larger SNR values, the system with the uniform power allocation outperforms the beamforming system. As expected, the optimal power allocation additionally increases the ASE. The optimal power allocation coefficients are given in Fig. 6, and it confirms that beamforming is optimal for $\mathrm{SNR}<8 \mathrm{~dB}$, and equal power

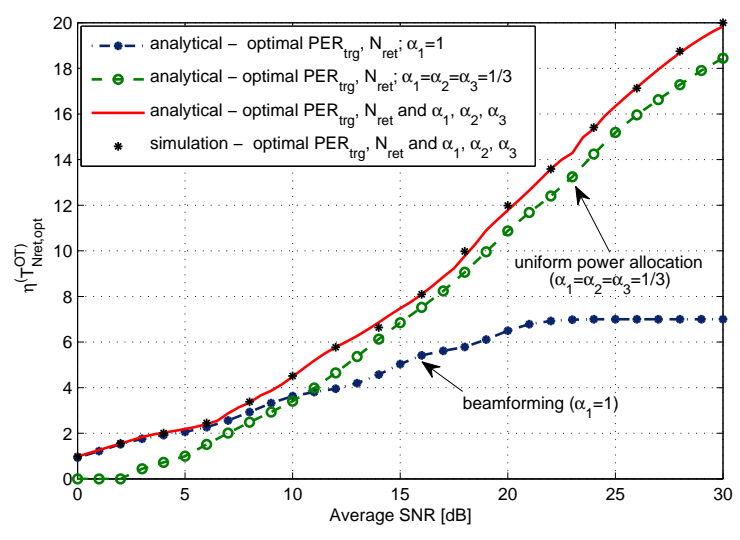

Fig. 5. Average spectral efficiency for MIMO-MB system with $N=M=3, p_{\text {loss, trg }}=0.01$, perfect CSI, PER trg $_{\text {and }}$ $N_{\text {ret }}$ optimally chosen, the impact of power allocation.

\begin{tabular}{|c|c|c|c|c|}
\hline$p_{\text {loss, } \operatorname{trg}}$ & $N_{\text {ret }}$ & $N=1, M=1$ & $N=2, M=2$ & $N=3, M=3$ \\
\hline \multirow{4}{*}{0.05} & 0 & $11-30$ & $5.5-30$ & $3.5-30$ \\
\cline { 2 - 5 } & 1 & $4-11$ & $1.5-5.5$ & $0-3.5$ \\
\cline { 2 - 5 } & 2 & $1.5-4$ & $0-1.5$ & - \\
\cline { 2 - 5 } & 3 & $0-1.5$ & - & - \\
\hline \multirow{4}{*}{0.0001} & 0 & - & - & $27.5-30$ \\
\cline { 2 - 5 } & 1 & - & $25.5-30$ & $22.5-27.5$ \\
\cline { 2 - 5 } & 2 & $21.5-30$ & $14-25.5$ & $10.5-22.5$ \\
\cline { 2 - 5 } & 3 & $0-21.5$ & $0-14$ & $0-10.5$ \\
\hline
\end{tabular}

Tab. 2. Optimal SNR ranges (given in $\mathrm{dB}$ ) for 0-3 retransmissions, various system dimensions and packet loss rates.

allocation in two stronger eigenchannels is nearly optimal for $7 \mathrm{~dB}<\mathrm{SNR}<19 \mathrm{~dB}$. The uniform power allocation (variable rate mode from [4]) is nearly optimal only in the narrow region $19 \mathrm{~dB}<\mathrm{SNR}<25 \mathrm{~dB}$. The fluctuations are the consequence of the fact that the constellations with the integer values of $m$ are used, and the transitions happen when a modulation format is changed. The limited number of constellations results in the saturation in two stronger eigenchannels for SNR $>25 \mathrm{~dB}$. Therefore, for very high SNR values, it is optimal to assign a large portion of the available power to the eigenchannel with the power gain determined by the smallest eigenvalue.

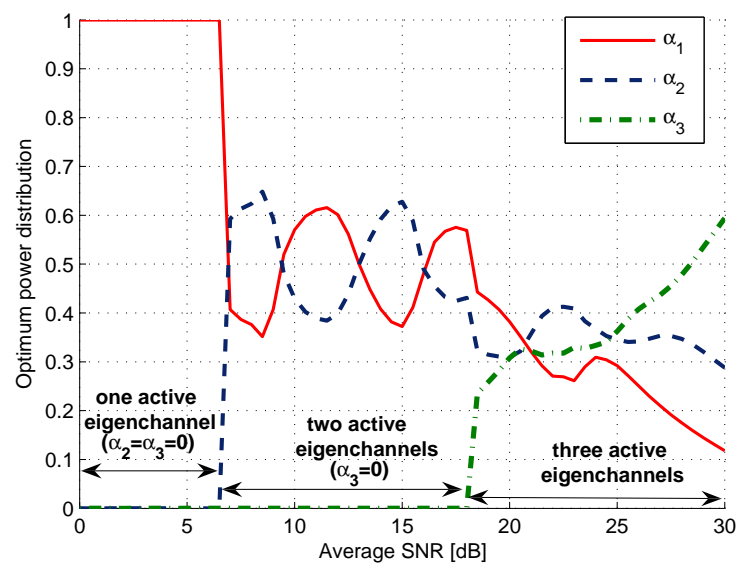

Fig. 6. Optimal power allocation coefficients for $N=M=3$, $p_{\text {loss, } \operatorname{trg}}=0.01$, perfect CSI. 

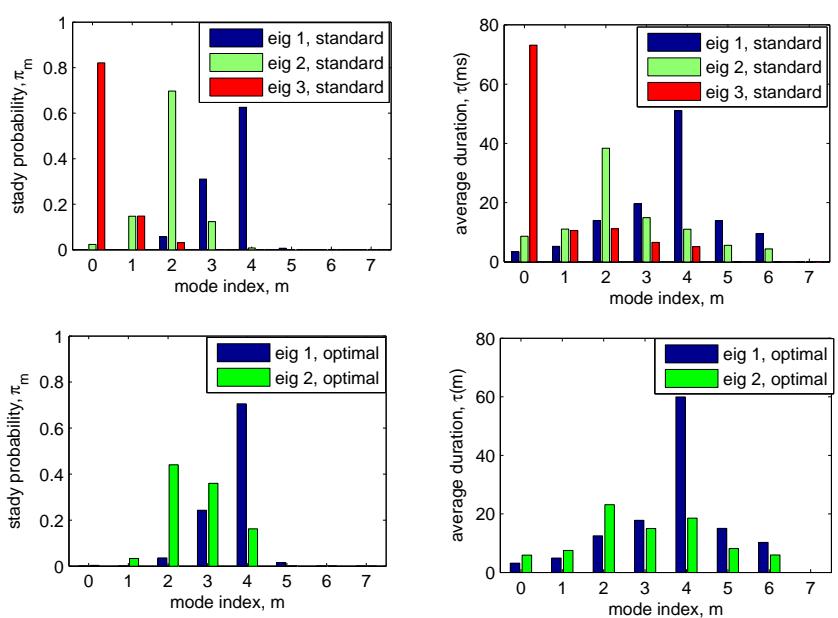

Fig. 7. Probability of mode choice and the average time that constellation is continuously used, $N=M=3, \bar{\gamma}=15 \mathrm{~dB}$, $f_{m}=11 \mathrm{~Hz}, p_{\text {loss, } \operatorname{trg}}=0.01$.

Numerical results for the probabilities of particular modes and the average time that a constellation is continuously used are calculated by using (10) and (16) and presented in Fig. 7. For typical values of maximal Doppler frequency and the frame duration $\left(f_{m}=11 \mathrm{~Hz}, T_{F}=2 \mathrm{~ms}\right)$, every particular constellation is continuously used during the transmission of the several transmission frames. For the case of system with $N=M=3$ and the uniform power allocation, in the strongest eigenchannel 16-QAM is chosen in approximately $62 \%$ of the transmitted frames and this constellation is continuously used during 25 successive frames. In the second eigenchannel, QPSK modulation is chosen in $65 \%$ of the transmitted frames and it continuously used during 20 successive frames on average. However, for the same case, the SNR in the third (weakest) eigenchannel is below the threshold for the successive transmission in about $80 \%$ time instants, and the third eigenchannel remains in outage state during 35 transmission frames in average. On the other hand, if we only apply optimal power allocation method described in this paper, transmission through the weakest eigenchannel is suspended till the moment when it enables reliable transmissions and power is reallocated to active eigenchannels. Therefore, there is no need to continuously send frames without payload in third eigenchannel and the optimal power allocation results in energy efficient transmission. Furthermore, the throughput in active eigenchannels is increased, the transmission modes with the higher spectral efficiency are chosen more frequently and more frames are continuously sent using the same modulation format. Therefore, the switching rate between the transmission modes in every particular eigenchannel is also reduced.

\section{Concluding Remarks}

In this paper, we have proposed an efficient cross-layer design, which combines the adaptive modulation with the truncated automatic repeat procedures in an arbitrary eigenchannel of MIMO system with multichannel beamforming. It has been shown that the proposed modification of the standard cross-layer design results in higher spectral efficiency and reduced delay, especially in high SNR regions. Although our modified design outperforms the standard method even for radio systems with one transmit and one receive antennas, we have further extended it to the eigenchannels of the MIMO-MB system. The corresponding expressions for the average spectral efficiency and the packet loss rate are derived in the closed form, for arbitrary diversity order. Also, we have derived the closed-form expression for the average time during which arbitrary particular constellation is continuously used.

The numerical optimization algorithm is applied to obtain the eigenchannel power allocation that additionally increases the spectral efficiency for the target packet error loss rate. It has been shown that the beamforming transmission is optimal for lower SNR values, and with the further increase of the SNR, power is reallocated to weaker eigenchannels. It has been shown that the average time during when one constellation is continuously used also depends on the power allocation. When an eigenchannel can not enable reliable transmission, it is turned off and the power is reallocated to the active eigenchannels. Using the optimal power allocation, the weakest eigenchannel is turned on only if the corresponding transmission satisfies QoS requirements. As a consequence, the overall average spectral efficiency and energy efficiency are increased, the maximal delay is reduced and the constellation switching rate in active eigenchannels is less frequent.

Finally, although the numerical optimization algorithm is not simple, it can be noticed that the proposed algorithm represents low-complex solution. The optimal power allocation coefficients, the optimal target packet error rate and the number of retransmissions depends on the average signal-tonoise ratio (link adaptation), not on the instantaneous SNR (as in the fast link adaptation). Therefore, the optimal parameters can be calculated for a typical range of average SNR values, with appropriate resolution, and stored in the look-up table. When the average SNR is detected, the optimum values of $N_{\text {ret }}$, the target PERs and the channel allocations coefficients for every particular eigenchannel are extracted from the table and the system performance can be determined by using the derived closed-form expressions (9)-(16).

\section{Appendix}

For completeness, we present here a brief description of the SQP optimization algorithm we use for obtaining the optimal ASE values. The SQP method represents the stateof-the-art in nonlinear programming methods [22] and it can be applied if we reorganize (20) as follows

$$
\min \{f(\mathbf{x})\}=\min \left\{-\eta\left(\operatorname{PER}_{\operatorname{trg}}^{(1)}, \ldots \operatorname{PER}_{\operatorname{trg}}^{(M)}, \alpha_{1}, \ldots \alpha_{M}\right)\right\},
$$


subject to:

$$
\begin{aligned}
& g_{0}(\mathbf{x})=\sum_{n=1}^{M} \alpha_{n}-1=0, \\
& g_{i}(\mathbf{x})=p_{\text {loss }}^{(i)}-p_{\text {loss, trg }}^{(i)} \leq 0, i=1, \ldots, M, \\
& g_{M+i}(\mathbf{x})=\mathrm{PER}_{\mathrm{trg}}^{(i)}-1 \leq 0, i=1, \ldots, M, \\
& g_{2 M+i}(\mathbf{x})=-\mathrm{PER}_{\mathrm{trg}}^{(i)} \leq 0, i=1, \ldots, M .
\end{aligned}
$$

The SQP optimization is performed for a constant number of retransmissions and a given CSI imperfection level. If we denote $\mathbf{x}=\left[\mathrm{PER}_{\mathrm{trg}}^{(1)}, \ldots \mathrm{PER}_{\mathrm{trg}}^{(M)}, \alpha_{1}, \ldots \alpha_{M}\right]$, the goal is to find a solution $\mathbf{x}^{*}$ that satisfies all KKT conditions [22] using Newton's iterative method:

$$
\mathbf{x}_{k+1}=\mathbf{x}_{k}+\alpha_{k} \mathbf{d}_{k},
$$

where $\mathbf{x}_{\mathbf{k}}$ represents a solution produced by the previous iteration, $\mathbf{d}_{k}$ is a search direction and is the value of adaptive step size. The iteration process is terminated when it is satisfied $\left\|\mathbf{x}_{k+1}-\mathbf{x}_{k}\right\| \leq \varepsilon$, where $\varepsilon$ represents predefined accuracy.

The principal idea of finding $\mathbf{d}_{k}$ is the formulation of a Quadratic Programming subproblem based on a quadratic approximation of the Lagrangian function at $\mathbf{x}_{k}$. Thus, the subproblem can be defined as

$$
\min f^{\prime}(\mathbf{d})=\frac{1}{2} \mathbf{d}^{T} \mathbf{H}_{k} \mathbf{d}+\nabla f\left(\mathbf{x}_{k}\right)^{T} \mathbf{d}
$$

subject to:

$$
\begin{aligned}
& \nabla g_{0}\left(\mathbf{x}_{k}\right)^{T} \mathbf{d}+g_{0}\left(\mathbf{x}_{k}\right)=0 \\
& \nabla g_{i}\left(\mathbf{x}_{k}\right)^{T} \mathbf{d}+g_{i}\left(\mathbf{x}_{k}\right) \leq 0, i=1, \ldots, 3 M,
\end{aligned}
$$

where $\mathbf{H}_{k}$ represents a positive definite approximation of the Hessian matrix of the Lagrangian function at $\mathbf{x}_{k}, \nabla$ is the differential operator and $T$ denotes the matrix transposition operator. The matrix $\mathbf{H}_{k}$ is updated in every iteration.

We used an active set method to solve the Quadratic Programming subproblem. It is also iterative algorithm with a prime task to find a set of active constrains (constrains associated with nonnegative Lagrange multipliers). For a initially assumed active set $\mathbf{W}_{0}$ and starting point $\mathbf{d}_{0}$, in the $n$th iteration algorithm finds $\mathbf{p}_{n}$ that minimizes $f^{\prime}\left(\mathbf{p}=\mathbf{d}-\mathbf{d}_{n}\right)$ over the active set $\mathbf{W}_{n}$. Then, a new value of potential solution $\mathbf{d}_{n+1}$ is calculated according to

$$
\mathbf{d}_{n+1}=\mathbf{d}_{n}+\beta_{n} \mathbf{p}_{n}
$$

where

$$
\beta_{n}=\min \left(1, \min _{i \notin W_{k}, \nabla g_{i}\left(\mathbf{x}_{k}\right)^{T} \mathbf{p}_{n}<0} \frac{g_{i}\left(\mathbf{x}_{k}\right)-\nabla g_{i}\left(\mathbf{x}_{k}\right)^{T} \mathbf{d}_{n}}{\nabla g_{i}\left(\mathbf{x}_{k}\right)^{T} \mathbf{p}_{n}}\right) .
$$

If $\mathbf{p}_{n}=0$ then Lagrange multipliers (denoted as $\left.\lambda=\left[\lambda_{0}, \lambda_{1} \ldots \lambda_{3 M}\right]\right)$ are calculated in order to examine if the
KKT condition are satisfied. If all $\lambda_{i} \geq 0, i \in W_{n} \cap I$, where $I$ is a set of inequality constraints, $\mathbf{d}_{n+1}$ is the solution of the subproblem optimization: $\mathbf{d}_{k}=\mathbf{d}_{n+1}$. But, if exists at least one $\lambda_{i}<0, i \in W_{n} \cap I$, then the constraint $j$, which corresponds to the smallest value of the Lagrange multiplier is removed from the active set $\left(W_{n+1}=W_{n} \backslash\{j\}\right)$ and optimization continues. If $\mathbf{p}_{n} \neq 0$ the solution is not yet found and the value of $\beta_{n}$ determines the next step. If $\beta_{n}<1$, the algorithm selects constraints that achieve the minimum in (A.5) and they are declared as the blocking constraints. Then, the new working set $\mathbf{W}_{n+1}$ is constructed by adding one of the blocking constraints to $\mathbf{W}_{k}$. If $\beta_{n}=1$ there are no new active constraints, the active set remains the same $\left(W_{n+1}=W_{n}\right)$ and the algorithm starts a new iteration. The value of adaptive step size $\alpha_{k}$ is evaluated in every iteration $k$ of the global quasi-Newton's method as a solution of one-dimensional line search of the merit function.

\section{Acknowledgments}

This work was supported by the Serbian Ministry of Science under project TR32007.

\section{References}

[1] TELATAR, I. E. Capacity of the multi antenna Gaussian channels. European Transactions on Telecommunications, 1999, vol. 10, p. 585-595. DOI: 10.1002/ett.4460100604

[2] GARTH, L. M., SMITH, P. J., SHAFI, M. Exact symbol error probabilities for SVD transmission of BPSK data over fading channels. In Proceedings of IEEE International Conference on Communications (ICC 2005). Seoul (South Korea), 2005, p. 2271-2276. ISBN: 0-7803-8938-7 DOI: 10.1109/ICC.2005.1494740

[3] ZHOU, Z., VUCETIC, B. Design of adaptive modulation using imperfect CSI in MIMO systems. IEE Electronic Letters, 2004, vol. 40, no. 17 , p. 1073-1075. ISSN: 0013-5194 DOI: 10.1049/el:20045077

[4] ZHOU, Z., VUCETIC, B., DOHLER, M., LI, Y. MIMO systems with adaptive modulation. IEEE Transactions on Vechicular Technology, 2005, vol. 54, no. 5, p. 1828-1842. ISSN: 0018-9545 DOI: 10.1109/TVT.2005.853886

[5] IVANIS, P., DRAJIC, D. Combined optimal power allocation and adaptive modulation for MIMO systems with imperfect CSI. In Proceedings of Telecommunications in Modern Satellite, Cable and Broadcasting Service (TELSIKS 2003). Nis (Serbia), 2004, p. 167170. ISBN: 0-7803-7963-2 DOI: 10.1109/TELSKS.2003.1246208

[6] NIKOLIC, B., DIMITRIJEVIC, B., MILOSEVIC, N., DJORDEVIC, G. T. Performance improvement of QPSK signal predetection EGC diversity receiver. Radioengineering, 2012, vol. 21, no. 3, p. 831-837.

[7] KANG, M, ALOUINI, MS. Largest Eigenvalue of Complex Wishart Matrices and Performance Analysis of MIMO MRC Systems. IEEE Journal of Selected Areas in Communications, 2003, vol. 21, no. 3, p. 418-431. ISSN: 0733-8716 DOI: 10.1109/JSAC.2003.809720

[8] ZHOU, S., GIANNAKIS, G.B. How Accurate Channel Prediction Needs to be for Transmit-Beamforming with Adaptive Modulation over Rayleigh MIMO Channels? IEEE Transactions on Wireless Communications, 2004, vol 3, no. 4, p. 1285-1294. ISSN: 536-1276 DOI: $10.1109 /$ TWC.2004.830842 
[9] IVANIS, P., DRAJIC, D., VUCETIC, B. Performance Evaluation of Adaptive MIMO-MRC Systems with Imperfect CSI by a Markov Model. In Proceedings of 65th IEEE Vechicular Technology Conference, VTC 2007, Dublin (Ireland), 2007, p. 1496-1500. ISSN: 1550-2252 DOI: 10.1109/VETECS.2007.312

[10] JIN, S, MCKAY, M.R., GAO, X., COLLINGS, I.B. MIMO multichannel beamforming: SER and outage using new eigenvalue distributions of complex noncentral Wishart matrices. IEEE Transactions on Communications, 2008, vol. 56, no. 3, p. 424-434. ISSN: 00906778 DOI: 10.1109/TCOMM.2008.060115

[11] IVANIS, P., DRAJIC, D., VUCETIC, B. Level crossing rates of Ricean MIMO channel eigenvalues for imperfect and outdated CSI IEEE Communications Letters, 2007, vol. 11, no. 10, p. 775-777. ISSN: 1089-7798 DOI: 10.1109/LCOMM.2007.070894

[12] LIU, Q, ZHOU, S, GIANNAKIS, G.B. Cross-layer combining of adaptive modulation and coding with truncated ARQ over wireless links. IEEE Transactions on Wireless Communications, 2004, vol. 3, no. 5, p. 1746-1755. ISSN: 1536-1276 DOI 10.1109/TWC.2004.833474

[13] MAAREF, A., AISSA, S. Combined Adaptive Modulation and Truncated ARQ for Packet Data Transmission in MIMO Systems. In Proceedings of IEEE Global Telecommunications Conference, Globecom 2004, Dallas (USA), 2004 p. 3818-38-22. ISBN: 0-7803-8794-5 DOI: 10.1109/GLOCOM.2004.1379083

[14] DANG, X., LIU, Y., YU, X. Performance analysis of cross-layer design with average PER constraint over MIMO fading channels. Taylor \& Francis International Journal of Electronics, 2015, vol. 102, no. 12 p. 2031-2045. DOI: 10.1080/00207217.2015.1018339

[15] YU, X., LIU, Y., LI, Y. ZHU, Q., YIN X., QIAN, K. Performance of cross-layer design with multiple outdated estimates in multiuser MIMO system. Radioengineering, 2014, vol. 23, no. 3, p. 863-871.

[16] FU, B., XIAO, Y., DENG, H., ZENG, H. A. Survey of cross-layer designs in wireless networks. IEEE Communications Surveys \& Tutorials, 2014, vol. 16, no. 1, p. 110-126. ISSN: 1553-877X DOI: 10.1109/SURV.2013.081313.00231

[17] JAFARI, A., MOHAMMADI, A. Cross layer design based on adaptive modulation and truncated ARQ in MIMO Rician channels. In Proceedings of International Symposium on Telecommunications (IST 2010). Tehran (Iran), 2010, p. 318-323. ISBN: 978-1-42448183-5 DOI: 10.1109/ISTEL.2010.5734044

[18] IVANIS, P. N., STOJNIC, M. M., DRAJIC, D. B. Exact bit error probabilities and packet error statistics for SVD transmission over timevarying dual-branch MIMO systems obtained by a Markov model. AEU - International Journal of Electronics and Communications, 2013, vol. 67, no. 2, p. 113-122. ISSN: 1434-8411

[19] ZANELLA, A., CHIANI, M. The PDF of the lth largest eigenvalue of central Wishart matrices and its application to the performance analysis of MIMO systems. In Proceedings of Global Telecommunications Conference (Globecom 2008). New Orleans (USA), 2008, p. 1063 1068. ISSN: 1930-529X DOI: 10.1109/GLOCOM.2008.ECP.210

[20] ABRAMOWITZ, M., STEGUN, I. A. Handbook of Mathematical Functions with Formulas, Graphs, and Mathematical Tables. New York: Dover Publications, 1972. ISBN: 0-486-61272-4
[21] GOLDSMITH, A. J., CHUA, S. Variable-rate variable-power MQAM for fading channels. IEEE Transactions on Communications, 1997, vol. 45, no. 10, p. 1218-1230. ISSN: 0090-6778 DOI: $10.1109 / 26.634685$

[22] BAZARAA, M. S., SHERALI, H. D., SHETTY, C. M. Nonlinear Programming Theory and Algorithms. New York: John Wiley, 2006. ISBN: 978-0-471-48600-8

[23] LI, Y., HUANG, X. The simulation of independent Rayleigh faders. IEEE Transactions on Communications, 2002, vol. 50, no. 9, p. 1503 1514. ISSN: 0090-6778 DOI: 10.1109/TCOMM.2002.802562

[24] YERUCHIM, M. C., BALABAN, P., SHANMUGAN, K. S. Simulation of Communication Systems. Dordrecht: Kluwer Academic Publishers, 2000. ISBN: 978-0-306-46971-8

\section{About the Authors...}

Predrag IVANIS was born in in Belgrade, Serbia. He received the B.Sc., M.Sc. and Ph.D. degrees in Electrical Engineering from School of Electrical Engineering, University of Belgrade, in 1999, 2004, and 2008, respectively. He is an associate professor at the Department of Telecommunications at the Faculty of Electrical Engineering, University of Belgrade. He has published over 50 scientific papers, and was the coauthor of one textbook. His research interests include wireless communications theory, information theory and error control coding.

Dejan DRAJIC was born in Belgrade, Serbia. He holds a Ph.D. degree (2004) in the area of chip equalization in advanced receivers for WCDMA downlink. His main research interests are future mobile networks (LTE and beyond LTE), M2M (Machine to Machine) and Internet of Things. From 2010 he is involved in FP7 projects related to these areas. Dejan has published more than 30 peer reviewed papers as well as 5 chapters in international Monographs. He is IEEE member and reviewer for some journals and conferences (Transactions on Emerging Telecommunications, Ad Hoc and Sensor Wireless Networks, etc.). Dejan holds a part time position at the School of Electrical Engineering, University of Belgrade, where he teaches M2M related courses.

Srdan BRKIC was born in Foca, Bosnia and Herzegovina. He received his M.Sc. in Electrical Engineering form School of Electrical Engineering, University of Belgrade, Serbia in 2010. He is currently finishing his $\mathrm{PhD}$ studies in the same university. His research interests include information theory, low-density parity-check coding, cognitive radio, and MIMO systems. 